九州大学学術情報リポジトリ

Kyushu University Institutional Repository

\title{
ON JACKKNIFE VARIANCE ESTIMATOR FOR KERNEL DENSITY ESTIMATOR AND ITS APPLICATION
}

Nishimoto, Atsufumi

Graduate School of Mathematics, Kyushu University

Maesono, Yoshihiko

Faculty of Mathematics, Kyushu University

https://doi.org/10.5109/1906479

出版情報: Bulletin of informatics and cybernetics. 47, pp.1-9, 2015-12. Research Association of Statistical Sciences

バージョン:

権利関係 : 


\title{
ON JACKKNIFE VARIANCE ESTIMATOR FOR KERNEL DENSITY ESTIMATOR AND ITS APPLICATION
}

\author{
By \\ Atsufumi Nishimoto* and Yoshihiko Maesono ${ }^{\dagger}$
}

\begin{abstract}
For a kernel estimator of a density function, we obtain an asymptotic representation of a jackknife variance estimator of the kernel density estimator and prove its consistency. Assuming a bandwidth $h_{n}=c n^{-\frac{1}{4}}(c>0)$, we also discuss an Edgeworth expansion with residual term $o\left(n^{-1 / 2}\right)$ and its validity. Many papers have studied theoretical properties of a kernel density estimator. Especially mean integrated squared errors are precisely studied. The asymptotic distribution of the estimator is also discussed, and it is easy to show asymptotic normality. In this paper, we will discuss higher order approximation of the distribution of the kernel estimator. We will obtain an Edgeworth expansion, which takes an explicit form with residual term $o\left(n^{-1 / 2}\right)$.
\end{abstract}

Key Words and Phrases: Kernel estimator, Density function, Edgeworth expansion, Jackknife variance estimator, Studentized estimator.

\section{Introduction}

Let $X_{1}, X_{2}, \cdots, X_{n}$ be independently and identically distributed (i.i.d.) random variables with distribution and density functions $F(x), f(x)$. The kernel type estimator of the density function $f$ is given by

$$
\hat{f}_{n}(x)=\frac{1}{n h_{n}} \sum_{i=1}^{n} K\left(\frac{x_{0}-X_{i}}{h_{n}}\right)
$$

where $h_{n}$ is a bandwidth parameter and $h_{n} \rightarrow 0, n h_{n} \rightarrow \infty(n \rightarrow \infty)$.K is a kernel function which satisfies

$$
\int_{-\infty}^{\infty} K(u) d u=1
$$

The kernel estimator was introduced by Fix and Hodges(1951) and Akaike(1954). Rosenblatt(1956) and Parzen(1962) have discussed basic properties of the estimator. Mean integrated squared errors of the kernel density estimator are discussed in many papers.

* Graduate School of Mathematics, Kyushu University, Motooka, Fukuoka 819-0395, Japan.

$\dagger$ Faculty of Mathematics, Kyushu University, Motooka, Fukuoka 819-0395, Japan. tel +81-92-8024480 maesono@math.kyushu-u.ac.jp 
There are also many papers which studied bias reduction, bandwidth selection, etc. It is easy to show the asymptotic normality of a standardized kernel estimator. Le us define

$$
\begin{aligned}
e_{1, n} & =E\left[\frac{1}{h_{n}} K\left(\frac{x_{0}-X_{1}}{h_{n}}\right)\right], \\
g_{1, n}(x) & =\frac{1}{h_{n}} K\left(\frac{x_{0}-X_{1}}{h_{n}}\right)-e_{1, n}, \\
e_{2, n} & =E\left[g_{1, n}^{2}\left(X_{1}\right)\right] .
\end{aligned}
$$

If $0<\int K^{2}(u) d u<\infty$ and $f^{\prime}(x)$ is bounded in a neighborhood around $x_{0}$, we have

$$
P\left(\frac{\sqrt{n}\left[\hat{f}_{n}\left(x_{0}\right)-f\left(x_{0}\right)\right]}{\sqrt{e_{2, n}}} \leq y\right)=\Phi(y)+o(1)
$$

where $\Phi(y)$ is the distribution function of the standard normal distribution $N(0,1)$.

For improvement of the normal approximation, Hall(1992) has discussed the Edgeworth expansion for the kernel density estimator. Umeno and Maesono(2013) have obtained the explicit form of the Edgeworth expansion and prove its validity. Hall(1992) has also discussed the Edgeworth expansion of the studentized kernel estimator, based on a naive estimator of the variance. In this paper we will obtain the asymptotic representation of the jackknife variance estimator of the kernel density estimator $\hat{f}_{n}\left(x_{0}\right)$, and show the consistency of the variance estimator. Using the asymptotic representation, we also get the Edgeworth expansion of a studentized kernel estimator. Here we discuss an explicit form of the expansion, and so we use the bandwidth with $h_{n}=c n^{-1 / 4}(c>0)$.

In Section 2, we will discuss the asymptotic properties of the jackknife variance estimator and the asymptotic normality. Using the asymptotic representation of the jackknife variance estimator, we obtain an asymptotic representation of the studenitized kernel estimator and its Edgeworth expansion with residual term $o\left(n^{-1 / 2}\right)$ in Section 3 and 4 .

\section{Jackknife variance estimator}

The jackknife variance estimator of $\hat{f}_{n}\left(x_{0}\right)$ is given by

$$
V_{J}=\frac{n-1}{n} \sum_{i=1}^{n}\left[\hat{f}_{n-1}^{(i)}\left(x_{0}\right)-\hat{f}_{n}\left(x_{0}\right)\right]^{2}
$$

where $\hat{f}_{n-1}^{(i)}\left(x_{0}\right)$ is a corresponding statistics based on the sample $X_{1}, \cdots, X_{i-1}, X_{i+1}, \cdots$, $X_{n}$ and, for the sake of simplicity, we use the same bandwidth $h_{n}$ for $\hat{f}_{n-1}^{(i)}\left(x_{0}\right)$. For this variance estimator, we have the following theorem.

Theorem 2.1. Assume that the kernel $K$ satisfies $\int u K(u) d u=0, \int u^{2} K(u) d u<$ $\infty, \int K^{2}(u) d u<\infty$, and $f^{\prime \prime}(x)$ is bounded in a neibourhoood of $x_{0}$. Then we have

$$
n V_{J}=e_{2, n}+\frac{2}{n} \sum_{i=1}^{n} H_{1, n}\left(X_{i}\right)+\frac{2}{n(n-1)} \sum_{C_{n, 2}} H_{2, n}\left(X_{i}, X_{j}\right)
$$


where

$$
\begin{aligned}
H_{1, n}(x) & =\frac{1}{2}\left[g_{1, n}^{2}(x)-e_{2, n}\right] \\
H_{2, n}(x, y) & =-g_{1, n}(x) g_{1, n}(y)
\end{aligned}
$$

Proof. Expanding the right hand side of (2), we will show (3). Since

$$
\hat{f}_{n-1}^{(i)}\left(x_{0}\right)=\frac{1}{(n-1)}\left\{\sum_{j=1}^{n} g_{1, n}\left(X_{j}\right)-g_{1, n}\left(X_{i}\right)\right\},
$$

we have

$$
\begin{aligned}
& \sum_{i=1}^{n}\left(\hat{f}_{n-1}^{(i)}\left(x_{0}\right)-\hat{f}_{n}\left(x_{0}\right)\right)^{2} \\
= & \frac{1}{(n-1)^{2}} \sum_{i=1}^{n} g_{1, n}^{2}\left(X_{j}\right)-\frac{1}{(n-1)^{2} n}\left\{\sum_{k=1}^{n} g_{1, n}\left(X_{k}\right)\right\}^{2} .
\end{aligned}
$$

Thus we can show that

$$
\begin{aligned}
& \frac{n-1}{n} \sum_{i=1}^{n}\left(\hat{f}_{n-1}^{(i)}\left(x_{0}\right)-\hat{f}_{n}\left(x_{0}\right)\right)^{2} \\
= & \frac{1}{n(n-1)} \sum_{i=1}^{n} g_{1, n}^{2}\left(X_{j}\right)-\frac{1}{(n-1) n^{2}}\left\{\sum_{k=1}^{n} g_{1, n}\left(X_{k}\right)\right\}^{2} .
\end{aligned}
$$

Thus we have the equation (3).

Let us consider approximations of $e_{1, n}$ and $e_{2, n}$. Using the transformation $u=$ $\left(x_{0}-X_{1}\right) / h_{n}$ and the Taylor expansion around $x_{0}$, we have

$$
\begin{aligned}
e_{1, n} & =\int K(u) f\left(x_{0}-h_{n} u\right) d u \\
& =f\left(x_{0}\right)+h_{n} f^{\prime}\left(x_{0}\right) \int u K(u) d u+\frac{1}{2} h_{n}^{2} f^{\prime \prime}\left(x_{0}\right) \int u^{2} K(u) d u+O\left(h_{n}^{3}\right), \\
e_{2, n} & =E\left[g_{1, n}^{2}\left(X_{1}\right)\right] \\
& =E\left[\left\{\frac{1}{h_{n}} K\left(\frac{x_{0}-X_{1}}{h_{n}}\right)-E\left[\frac{1}{h_{n}} K\left(\frac{x_{0}-X_{1}}{h_{n}}\right)\right]\right\}^{2}\right] \\
& =\frac{1}{h_{n}} \int K^{2}(u) f\left(x_{0}-h_{n} u\right) d u-\left\{E\left[\frac{1}{h_{n}} K\left(\frac{x_{0}-X_{i}}{h_{n}}\right)\right]\right\}^{2} \\
= & \frac{1}{h_{n}} \int K^{2}(u)\left\{f\left(x_{0}\right)-h_{n} u f^{(1)}\left(x_{0}\right)+\frac{1}{2}\left(h_{n} u\right)^{2} f^{\prime \prime}\left(x_{0}\right)\right\} d u \\
& -f^{2}\left(x_{0}\right)+O\left(h_{n}^{2}\right) .
\end{aligned}
$$

Similarly we can show that

$$
E\left(g_{1, n}^{k}\left(X_{1}\right)\right)=\frac{1}{h_{n}^{k-1}} f\left(x_{0}\right) \int K^{k}(u) d u+O\left(h_{n}^{-k+2}\right)
$$


Since $e_{2, n}=O\left(h_{n}^{-1}\right)=O\left(n^{1 / 4}\right)$, let us define

$$
\sigma_{n}^{2}=h_{n} e_{2, n} .
$$

Therefore we consider

$$
n^{3 / 4} V_{J}=\sigma_{n}^{2}+B_{1, n}+B_{2, n}
$$

where

$$
B_{1, n}=2 n^{-5 / 4} \sum_{i=1}^{n} H_{1, n}\left(X_{i}\right) \quad \text { and } \quad B_{2, n}=2 n^{-5 / 4}(n-1)^{-1} \sum_{1 \leq i<j \leq n} H_{2, n}\left(X_{i}, X_{j}\right) .
$$

Using the moment evaluations, we can show the following theorem.

TheOrem 2.2. Let us assume that $0<\int u^{2} K(u) d u<\infty$ and $f^{\prime \prime}(x)$ is bounded in the neighborhood of $x_{0}$, we have

$$
\begin{aligned}
& \left|n^{3 / 4} V_{J}-\sigma_{n}^{2}\right| \stackrel{P}{\longrightarrow} 0, \\
& \frac{\hat{f}_{n}\left(x_{0}\right)-E\left[\hat{f}_{n}\left(x_{0}\right)\right]}{\sqrt{V_{J}}} \stackrel{L}{\longrightarrow} G
\end{aligned}
$$

where $G$ is $N(0,1)$.

Proof. Since $H_{1, n}\left(X_{i}\right), H_{2, n}\left(X_{i}, X_{j}\right)$ are $H$-decomposition, we have moment evaluations of them. Using the moment evaluations, the equation (6) and the Markov inequality, we can show that

$$
\begin{aligned}
& E\left(B_{1, n}^{2}\right)=4 n^{-5 / 2} n E\left[H_{1, n}^{2}\left(X_{i}\right)\right]=O\left(n^{-3 / 4}\right), \\
& E\left(B_{2, n}^{2}\right) \leq n^{-\frac{9}{2}} n^{2} E\left[H_{1, n}^{2}\left(X_{i}\right)\right] E\left[H_{1, n}^{2}\left(X_{j}\right)\right]=O\left(n^{-2}\right) .
\end{aligned}
$$

Thus we have the desired result.

\section{Studentized kernel estimator}

Using the asymptotic representation of the jackknife variance estimator, we obtain an asymptotic representation of the studentized kernel estimator

$$
\frac{\hat{f}_{n}\left(x_{0}\right)-E\left[\hat{f}_{n}\left(x_{0}\right)\right]}{\sqrt{V_{J}}} .
$$

Hereafter, we use a symbol $o_{L}\left(n^{-1 / 2}\right)$ which satisfies

$$
P\left(\left|o_{L}\left(n^{-1 / 2}\right)\right| \geq n^{-1 / 2}(\log n)^{-1}\right)=o\left(n^{-1 / 2}\right) .
$$

Thus we can ignore $o_{L}\left(n^{-1 / 2}\right)$ when we discuss the Edgeworth expansion with residual term $o\left(n^{-1 / 2}\right)$. Using the Markov inequality, we can show that if $E\left(\left|R_{n}\right|^{c}\right)=$ $O\left(n^{-1 / 2-c / 2-\delta}\right)(c>0, \delta>0)$, we have $R_{n}=o_{L}\left(n^{-1 / 2}\right)$. Let us define a standardized $g_{1, n}$ and its summation

$$
\begin{aligned}
& g_{1, n}^{*}(x)=e_{2, n}^{-1 / 2} g_{1, n}(x)=h_{n}^{1 / 2} \sigma_{n} g_{1, n}(x), \\
& A_{1, n}=\frac{1}{n} \sum_{i=1}^{n} g_{1, n}^{*}\left(X_{i}\right) .
\end{aligned}
$$


Further let us define

$$
\begin{aligned}
\zeta= & n^{-1 / 4} E\left[g_{1, n}^{*}\left(X_{1}\right) H_{1, n}\left(X_{1}\right)\right], \\
\tilde{\alpha}_{1, n}(x)= & n^{-1 / 4} g_{1, n}^{*}(x) H_{1, n}(x)-\zeta, \\
\alpha_{2, n}(x, y)= & n^{-1 / 4}\left\{g_{1, n}^{*}(x) H_{1, n}(y)+g_{1, n}^{*}(y) H_{1, n}(x)\right\}, \\
\alpha_{3, n}(x, y, z)= & n^{-1 / 2}\left\{g_{1, n}^{*}(x) H_{1, n}(y) H_{1, n}(z)+g_{1, n}^{*}(y) H_{1, n}(x) H_{1, n}(z)\right\} \\
& +g_{1, n}^{*}(z) H_{1, n}(x) H_{1, n}(y) .
\end{aligned}
$$

Then we have an asymptotic representation of the studentized kernel density estimator as follows.

THEOREM 3.1. Assume that the kernel $K$ is a bounded function and satisfies $\int u K(u) d u=$ $0, \int u^{2} K(u) d u<\infty$. If $f^{\prime \prime}(x)$ is bounded in a neighborhood of $x_{0}$, we have

$$
\frac{\hat{f}_{n}\left(x_{0}\right)-E\left[\hat{f}_{n}\left(x_{0}\right)\right]}{\sqrt{V_{J}}}=\sqrt{n} U_{n}\left(x_{0}\right)-\frac{\zeta}{\sqrt{n} \sigma_{n}^{3}}+o_{L}\left(n^{-1 / 2}\right)
$$

where

$$
\begin{aligned}
& U_{n}\left(x_{0}\right) \\
& =A_{1, n}+\frac{1}{n^{2} \sigma_{n}^{2}} \sum_{i=1}^{n} \tilde{\alpha}_{1, n}\left(X_{i}\right)-\frac{1}{n^{2} \sigma_{n}^{2}} \sum_{1 \leq i<j \leq n} \alpha_{2, n}\left(X_{i}, X_{j}\right) \\
& +\frac{3}{n^{3} \sigma_{n}^{4}} \sum_{1 \leq i<j<k \leq n} \alpha_{3, n}\left(X_{i}, X_{j}, X_{k}\right)+\frac{\zeta}{n} .
\end{aligned}
$$

PRoOF. Using the Taylor expansion around $\sigma_{n}^{2}$, we have

$$
\frac{1}{\sqrt{n^{3 / 4} V_{J}}}=\frac{1}{\sigma_{n}}-\frac{1}{2 \sigma_{n}^{3}}\left(B_{1, n}+B_{2, n}\right)+\frac{3}{8 \sigma_{n}^{5}}\left(B_{1, n}+B_{2, n}\right)^{2}+O\left(\left(B_{1, n}+B_{2, n}\right)^{3}\right) .
$$

It follows from the Hoeffding(1961) decomposition and moment evaluations that

$$
E\left|B_{1, n} B_{2, n}\right| \leq\left\{E\left(B_{1, n}^{2}\right) E\left(B_{2, n}^{2}\right)\right\}^{1 / 2}=O\left(n^{-3 / 8-1}\right)=O\left(n^{-1 / 2-1 / 2-3 / 8}\right),
$$

and then $B_{1, n} B_{2, n}=o_{L}\left(n^{-1 / 2}\right)$. Similarly $B_{2, n}^{2}$ and $\left(B_{1, n}+B_{2, n}\right)^{3}$ are $o_{L}\left(n^{-1 / 2}\right)$, and SO

$$
\frac{1}{\sqrt{n^{3 / 4} V_{J}}}=\frac{1}{\sigma_{n}}-\frac{1}{2 \sigma_{n}^{3}}\left(B_{1, n}+B_{2, n}\right)+\frac{3}{8 \sigma_{n}^{5}} B_{1, n}^{2}+o_{L}\left(n^{-1 / 2}\right) .
$$

Next we will evaluate products with $A_{1, n}$. Since

$$
\begin{gathered}
\left.\begin{array}{l}
A_{1, n} B_{2, n} \\
=
\end{array} n^{-23 / 8}\right) \sum_{1 \leq i<j \leq n}\left\{g_{1, n}\left(X_{i}\right) H_{2, n}\left(X_{i}, X_{j}\right)+g_{1, n}\left(X_{j}\right) H_{2, n}\left(X_{i}, X_{j}\right)\right\} \\
+O\left(n^{-23 / 8}\right) \sum_{1 \leq i<j \leq k \leq n}\left\{g_{1, n}\left(X_{i}\right) H_{2, n}\left(X_{j}, X_{k}\right)+g_{1, n}\left(X_{j}\right) H_{2, n}\left(X_{i}, X_{k}\right)\right. \\
\left.+g_{1, n}\left(X_{k}\right) H_{2, n}\left(X_{i}, X_{j}\right)\right\},
\end{gathered}
$$


using the Hoeffding decomposition and moment evaluations, it is easy to show that these terms are $o_{L}\left(n^{-1 / 2}\right)$. Also we get

$$
\begin{aligned}
& A_{1, n} B_{1, n} \\
= & \frac{1}{n^{9 / 4} \sigma_{n}^{2}} \sum_{i=1}^{n}\left\{g_{1, n}^{*}\left(X_{i}\right) H_{1, n}\left(X_{i}\right)-E\left[g_{1, n}^{*}\left(X_{1}\right) H_{1, n}\left(X_{1}\right)\right]\right\} \\
& +\frac{1}{n^{5 / 4} \sigma_{n}^{2}} E\left[g_{1, n}^{*}\left(X_{1}\right) H_{1, n}\left(X_{1}\right)\right] \\
& -\frac{1}{n^{9 / 4} \sigma_{n}^{2}} \sum_{1 \leq i<j \leq n}\left\{g_{1, n}^{*}\left(X_{i}\right) H_{1, n}\left(X_{j}\right)+g_{1, n}^{*}\left(X_{j}\right) H_{1, n}\left(X_{i}\right)\right\} .
\end{aligned}
$$

Finally we can show that

$$
\begin{aligned}
& \frac{A_{1, n} B_{1, n}^{2}}{n^{7 / 2} \sigma_{n}^{4}} \sum_{1 \leq i<j<k \leq n}\left\{g_{1, n}^{*}\left(X_{i}\right) H_{1, n}\left(X_{j}\right) H_{1, n}\left(X_{k}\right)+g_{1, n}^{*}\left(X_{j}\right) H_{1, n}\left(X_{i}\right) H_{1, n}\left(X_{k}\right)\right. \\
& \left.+g_{1, n}^{*}\left(X_{k}\right) H_{1, n}\left(X_{i}\right) H_{1, n}\left(X_{j}\right)\right\}+o_{L}\left(n^{-1 / 2}\right) .
\end{aligned}
$$

\section{Edgeworth expansion}

Using the asymptotic representation (7), we obtain the Edgeworth expansion with residual term $o\left(n^{-1 / 2}\right)$. Let us define

$$
\begin{aligned}
\zeta= & E\left[g_{1, n}^{*}\left(X_{1}\right) H_{1, n}\left(X_{1}\right)\right], \\
\kappa_{3}= & E\left[\left\{g_{1, n}^{*}\left(X_{1}\right)\right\}^{3}\right]+3 E\left[g_{1, n}^{*}\left(X_{1}\right) g_{1, n}^{*}\left(X_{2}\right) \alpha_{2, n}\left(X_{1}, X_{2}\right)\right], \\
\kappa_{4}= & E\left[\left\{g_{1, n}^{*}\left(X_{1}\right)\right\}^{4}\right]-3+4 E\left[g_{1, n}^{*}\left(X_{1}\right) g_{1, n}^{*}\left(X_{2}\right) g_{1, n}^{*}\left(X_{3}\right) \alpha_{3, n}\left(X_{1}, X_{2}, X_{3}\right)\right] \\
& +12 E\left[\left\{g_{1, n}^{*}\right\}^{2}\left(X_{1}\right) g_{1, n}^{*}\left(X_{2}\right) \alpha_{2, n}\left(X_{1}, X_{2}\right)\right] \\
& +12 E\left[g_{1, n}^{*}\left(X_{1}\right) g_{1, n}^{*}\left(X_{2}\right) \alpha_{2, n}\left(X_{1}, X_{3}\right) \alpha_{2, n}\left(X_{2}, X_{3}\right)\right], \\
P_{1}(y)= & \frac{\kappa_{3}\left(y^{2}-1\right)}{6}, \\
P_{2}(y)= & \left\{n^{1 / 4} \zeta+\frac{E\left[\alpha_{2, n}^{2}\left(X_{1}, X_{2}\right)\right]}{4}\right\} y+\frac{\kappa_{4}}{24}\left(y^{3}-3 y\right)+\frac{\kappa_{3}^{2}}{72}\left(y^{5}-10 y^{3}+15 y\right) .
\end{aligned}
$$

Using the Edgeworth expansion of Lai and Wang(1993), it is easy to show that

$$
\begin{aligned}
& P\left\{\frac{\hat{f}\left(x_{0}\right)-E\left[\hat{f}\left(x_{0}\right)\right]}{\sqrt{V_{J}}} \leq y\right\} \\
= & \Phi(y)-n^{-1 / 2} \phi(y) P_{1}(y)-n^{-1} \phi(y) P_{2}(y)+o\left(n^{-1 / 2}\right) .
\end{aligned}
$$

The validity of the Edgeworth expansion (8) can be proved by using the method of García-Soidán et al.(1997). Let us evaluate each term. Note that we assume the kernel $K(\cdot)$ is symmetric around 0 . Using the transformation and the Taylor expansion, it is 
easy to see that

$$
\begin{aligned}
E\left[\left\{g_{1, n}^{*}\left(X_{1}\right)\right\}^{3}\right] & =e_{2, n}^{-3 / 2} E\left[\frac{1}{h_{n}^{3}} K^{3}\left(\frac{x_{0}-X_{1}}{h_{n}}\right)\right]+O\left(h_{n}^{1 / 2}\right) \\
& =\frac{1}{h_{n}^{2} e_{2, n}^{3 / 2}} f\left(x_{0}\right) \int_{-\infty}^{\infty} K^{3}(u) d u+O\left(h_{n}^{1 / 2}\right) \\
& =\frac{1}{h_{n}^{1 / 2} \sigma_{n}^{3}} f\left(x_{0}\right) \int_{-\infty}^{\infty} K^{3}(u) d u+O\left(h_{n}^{1 / 2}\right) .
\end{aligned}
$$

Similarly we can show that

$$
\begin{aligned}
& E\left[g_{1, n}^{*}\left(X_{1}\right) g_{1, n}^{*}\left(X_{2}\right) \alpha_{2, n}\left(X_{1}, X_{2}\right)\right] \\
= & n^{-1 / 4} E\left[\left\{g_{1, n}\left(X_{1}\right)\right\}^{2}\right] E\left[g_{1,2}^{*} H_{1, n}\left(X_{1}\right)\right] \\
= & \frac{1}{n^{1 / 4} h_{n}^{3 / 2} \sigma_{n}^{3}} f\left(x_{0}\right) \int_{-\infty}^{\infty} K^{3}(u) d u+O\left(n^{-1 / 4} h_{n}^{-1 / 2}\right) .
\end{aligned}
$$

Furthermore, using the same method, we get

$$
\begin{aligned}
& E\left[\left\{g_{1, n}^{*}\left(X_{1}\right)\right\}^{4}\right]=O\left(n^{1 / 4}\right), \\
& E\left[g_{1, n}^{*}\left(X_{1}\right) g_{1, n}^{*}\left(X_{2}\right) g_{1, n}^{*}\left(X_{3}\right) \alpha_{3, n}\left(X_{1}, X_{2}, X_{3}\right)\right]=O\left(n^{1 / 4}\right), \\
& E\left[g_{1, n}^{*}\left(X_{1}\right) g_{1, n}^{*}\left(X_{2}\right) \alpha_{2, n}\left(X_{1}, X_{3}\right) \alpha_{2, n}\left(X_{2}, X_{3}\right)\right]=O\left(n^{-1 / 2} h_{n}^{-2}\right), \\
& E\left[g_{1, n}^{*}\left(X_{1}\right) g_{1, n}^{*}\left(X_{2}\right) \alpha_{2, n}\left(X_{1}, X_{3}\right) \alpha_{2, n}\left(X_{2}, X_{3}\right)\right]=O\left(n^{-1 / 2} h_{n}^{-3}\right), \\
& E\left[\alpha_{2, n}^{2}\left(X_{1}\right)\right]=O\left(n^{-1 / 2} h_{n}^{-3}\right)
\end{aligned}
$$

The coefficient of these values are $n^{-1}$, and so we can ignore these terms. Finally we have

$$
\begin{aligned}
& n^{-3 / 4} E\left[g_{1, n}^{*}\left(X_{1}\right) H_{1, n}\left(X_{1}\right)\right]=O\left(n^{-1} h_{n}^{-3 / 2}\right), \\
& E\left[\alpha_{2, n}^{2}\left(X_{1}, X_{2}\right)\right]=O\left(n^{-1 / 2} h_{n}^{-3}\right) .
\end{aligned}
$$

Then we have the following theorem.

THEOREM 4.1. Assume that the kernel $K$ is a bounded function and satisfies $\int u K(u) d u=$ $0, \int u^{2} K(u) d u<\infty$. If $f^{\prime \prime}(x)$ is bounded in a neighborhood of $x_{0}$, we have

$$
\begin{aligned}
& P\left\{\frac{\hat{f}\left(x_{0}\right)-E\left[\hat{f}\left(x_{0}\right)\right]}{\sqrt{V_{J}}} \leq y\right\} \\
= & \Phi(y)-n^{-1 / 2} \phi(y) \frac{y^{2}-1}{6 \sigma_{n}^{3}} f\left(x_{0}\right) \int_{-\infty}^{\infty} K^{3}(u) d u\left\{h_{n}^{-1 / 2}+n^{1 / 4} h_{n}^{-3 / 2}\right\}+o\left(n^{-1 / 2}\right) .
\end{aligned}
$$

The bias $E\left[\hat{f}\left(x_{0}\right)\right]-f\left(x_{0}\right)$ is not ignorable. We assume that $f^{(4)}(x)$ is bounded around $x_{0}$. Since $\int u K(u) d u=\int u^{3} K(u) d u=0$, we can obtain the approximation of the bias

$$
\begin{aligned}
& E\left[\frac{1}{h_{n}} K\left(\frac{x_{0}-X_{1}}{h_{n}}\right)\right]-f\left(x_{0}\right) \\
= & \frac{n^{3 / 8} h_{n}^{2}}{2} \int_{-\infty}^{\infty} u^{2} K(u) d u+O\left(n^{3 / 8} h_{n}^{4}\right) .
\end{aligned}
$$


Using the probability evaluation of the large deviation for $U$-statistics (Malevich and Abdalimov(1979)), we have

$$
n^{3 / 8} h_{n}^{2}\left(B_{1, n}+B_{2, n}\right)=o_{L}\left(n^{-1 / 2}\right)
$$

and then

$$
\frac{E\left[g_{1, n}\left(X_{1}\right)\right]-f\left(x_{0}\right)}{\sqrt{V_{J}}}=b_{n}+o_{L}\left(n^{-1 / 2}\right)
$$

where

$$
b_{n}=\frac{n^{3 / 8} h_{n}^{2}}{2 \sigma_{n}} f^{\prime \prime}\left(x_{0}\right) \int_{-\infty}^{\infty} u^{2} K(u) d u .
$$

Since $b_{n}=O\left(n^{-1 / 8}\right.$, we have

$$
\begin{aligned}
\Phi\left(y+b_{n}\right)= & \Phi(y)+b_{n} \phi(y)-\frac{b_{n}^{2}}{2} y \phi(y)+\frac{b_{n}^{3}\left(y^{2}-1\right)}{6} \phi(y) \\
& -\frac{b_{n}^{4}\left(y^{3}-3 y\right)}{24} \phi(y)+o\left(n^{-1 / 2}\right) .
\end{aligned}
$$

Finally, we can get the following theorem.

THEOREM 4.2. Assume that the kernel $K$ is a bounded function and satisfies $\int u K(u) d u=$ $0, \int u^{2} K(u) d u<\infty$. If $f^{(4)}(x)$ is bounded in a neighborhood of $x_{0}$, we have

$$
\begin{aligned}
& P\left\{\frac{\hat{f}\left(x_{0}\right)-E\left[\hat{f}\left(x_{0}\right)\right]}{\sqrt{V_{J}}} \leq y\right\} \\
= & \Phi(y)-n^{-1 / 2} \phi(y) \frac{y^{2}-1}{6 \sigma_{n}^{3}} f\left(x_{0}\right) \int_{-\infty}^{\infty} K^{3}(u) d u\left\{h_{n}^{-1 / 2}+n^{1 / 4} h_{n}^{-3 / 2}\right\} \\
& +b_{n} \phi(y)-\frac{b_{n}^{2}}{2} y \phi(y)+\frac{b_{n}^{3}\left(y^{2}-1\right)}{6} \phi(y)-\frac{b_{n}^{4}\left(y^{3}-3 y\right)}{24} \phi(y)+o\left(n^{-1 / 2}\right) .
\end{aligned}
$$

\section{Acknowledgement}

This research was supported by JSPS Grant-in-Aid for Exploratory Research No.15K11995.

\section{References}

Akaike, H. (1954). An approximation to the density function. Ann. Inst. Statist. Math. $6,127-132$

Fix, E. and Hodges, J.L. (1951). Discriminatory analysis nonparametric discrimination: consistency properties. Report No.4, Project no.21-29-004

Hall, P. (1992). The Bootstrap and Edgeworth Expansion. Springer-Verlag, New York.

García-Soidán, P.H., González-Manteiga W. and Prada-Sánchez, J.M. (1997).

Edgeworth expansions for nonparametric distribution estimation with applications. Jour. Stat. Plann. Inf. 65, 213-231 
Hoeffiding, W. (1961). The strong law of large numbers for U-statistics.Univ. of North Carolina Institute of statistics. Mimeo Series.No.302

Lai, T.L. and Wang, J.Q. (1993). Edgeworth expansion for symmetric statistics with applications to bootstrap methods. Statistica Sinica, 3, 517-542

Malevich, T.L. and Abdalimov B. (1979). Large deviation probabilities for $U$-statistics. Theory of Probab. Appl., 24, 215-219

Parzen, E. (1962). On the estimation of a probability density function and the mode.Ann. Math. Statist. 33, 1065-1076

Rosenblatt, M. (1956). Remarks on some nonparametric estimates of a density function.Ann. Math. Statist. 27, 832-837

Umeno, S. and Maesono, Y. (2013). Improvement of normal approximation for kernel density estimator. Bulletin of Informatics and Cybernetics 45, 11-24

Received December 15, 2014

Revised March 20, 2015 